Louisiana State University

LSU Digital Commons

Faculty Publications

Department of Biological Sciences

8-1-2007

\title{
The katanin microtubule severing protein in plants
}

David H. Burk

University of Georgia

Ruiqin Zhong

University of Georgia

Zheng Hua Ye

University of Georgia

Follow this and additional works at: https://digitalcommons.Isu.edu/biosci_pubs

Recommended Citation

Burk, D., Zhong, R., \& Ye, Z. (2007). The katanin microtubule severing protein in plants. Journal of Integrative Plant Biology, 49 (8), 1174-1182. https://doi.org/10.1111/j.1672-9072.2007.00544.x

This Article is brought to you for free and open access by the Department of Biological Sciences at LSU Digital Commons. It has been accepted for inclusion in Faculty Publications by an authorized administrator of LSU Digital Commons. For more information, please contact ir@lsu.edu. 
.Invited Review.

\title{
The Katanin Microtubule Severing Protein in Plants
}

\author{
David H. Burk*, Ruiqin Zhong and Zheng-Hua Ye** \\ (Department of Plant Biology, University of Georgia, Athens, Georgia 30602, USA)
}

\begin{abstract}
Katanin is a heterodimeric microtubule (MT) severing protein that uses energy from ATP hydrolysis to generate internal breaks along MTs. Katanin p60, one of the two subunits, possesses ATPase and MT-binding/severing activities, and the p 80 subunit is responsible for targeting of katanin to certain subcellular locations. In animals, katanin plays an important role in the release of MTs from their nucleation sites in the centrosome. It is also involved in severing MTs into smaller fragments which can serve as templates for further polymerization to increase MT number during meiotic and mitotic spindle assembly. Katanin homologs are present in a wide variety of plant species. The Arabidopsis katanin homolog has been shown to possess ATP-dependent MT severing activity in vitro and exhibit a punctate localization pattern at the cell cortex and the perinuclear region. Disruption of katanin functions by genetic mutations causes a delay in the disappearance of the perinuclear MT array and results in an aberrant organization of cortical MTs in elongating cells. Consequently, katanin mutations lead to defects in cell elongation, cellulose microfibril deposition, and hormonal responses. Studies of katanin in plants provide new insights into our understanding of its roles in cellular functions.
\end{abstract}

Key words: katanin; microtubules; microtubule severing protein.

Burk DH, Zhong R, Ye Z-H (2007). The katanin microtubule severing protein in plants. J. Integr. Plant. Biol. 49(8), 1174-1182.

Available online at www.blackwell-synergy.com/links/toc/jipb, www.jipb.net

The microtubule (MT) cytoskeleton plays important roles in diverse cellular processes in plants, such as mitosis, meiosis, organelle transport, cell morphogenesis, and cellulose microfibril deposition. MTs undergo dynamic reorganizations during progression of the cell cycle to form four distinct arrays, including preprophase band, mitotic spindle, phragmoplast, and cortical MTs (Baskin 2000). In each array, the MTs exhibit dynamic changes through a treadmilling mechanism in which the plus end undergoes polymerization-biased dynamic instability and the minus end displays slow depolymerization (Ehrhardt and Shaw 2006). It has been shown that new MTs are continuously polymerized at the nucleation sites and then released and

Received 3 Apr. 2007 Accepted 18 Apr. 2007

Supported by a grant from the US Department of Energy, Bioscience Division (DE-FG02-03ER15 415).

*Current address: Pennington Biomedical Research Center, Louisiana State

University, Baton Rouge, LA 70808, USA

**Author for correspondence.

Tel: +1706 542 1832;

Fax: +1706 5421805

E-mail: <zhye@plantbio.uga.edu >.

(C) 2007 Institute of Botany, the Chinese Academy of Sciences

doi: 10.1111/j.1672-9072.2007.00544.x translocated to form distinct structures (Ehrhardt and Shaw 2006). Although the mechanism responsible for MT release from the nucleation sites is not known, a severing activity is likely involved in freeing the MT minus ends from their attachments to the nucleation sites. In animals, the katanin MT severing protein has been shown to be required for the release of MTs from the nucleation sites in the centrosome (Ahmad et al. 1999; Karabay et al. 2004). Katanin also severs MTs along their length and the fragmented MTs can serve as templates for further MT polymerization, which provides another mechanism for MT assembly in addition to the $\gamma$-tubulin-dependent nucleation (McNally etal. 2006). Katanin homologs are known to exist in plants and they have been demonstrated to play essential roles in cortical MT organization and many MT-regulated cellular processes (Bichet et al. 2001; Burk et al. 2001; McClinton et al. 2001; Burk and Ye 2002; Stoppin-Mellet et al. 2002; Webb et al. 2002; Bouquin et al. 2003). In this article, we present a review on studies of the katanin MT severing protein with a focus on its roles in plants.

\section{Discovery of the Katanin MT Severing Protein}

Proteins with MT severing activity were first discovered in Xenopus egg extracts by Vale (1991). It was proposed that, 
like the disassembly of the actin cytoskeleton that is aided by proteins that sever filamentous actin, the disassembly of MTs might also be stimulated by severing proteins (in addition to their intrinsic dynamic properties, such as dynamic instability). To identify MT severing activity, Vale (1991) treated taxolstabilized, rhodamine-labeled MTs with extracts from Xenopus eggs that were in the mitotic state. The taxol-stabilized, fluorescent MTs were first immobilized on the surface of a glass coverslip. On incubation with the Xenopus egg mitotic extracts, the MTs were fragmented within minutes, suggesting that the mitotic extracts contain MT severing activity that can disassemble MTs along their length. The severing activity was further proven to involve a protein or proteins because it was inactivated by trypsin protease or heat treatment of the mitotic extracts.

After the detection of MT severing activity in Xenopus mitotic extracts, McNally and Vale (1993) set out to purify and characterize the protein(s) responsible. Using sea urchin egg extracts as a source, they identified two co-purified polypeptides with molecular weights of $60 \mathrm{kD}$ (p60) and $80 \mathrm{kD}$ (p80) that are associated with the MT severing activity. This heterodimeric protein was shown to able to sever and disassemble taxolstabilized MTs in the presence of ATP (Figure 1A-D), and hence was named katanin after katana, the Japanese word for samurai sword. Katanin was further shown to possess ATPase activity that is stimulated by MTs.

\section{Biochemical Properties of Katanin}

The molecular identity and biochemical properties of the two katanin subunits p60 and p80 were first revealed after the cloning of their cDNAs from sea urchin (Hartman et al. 1998). Analysis of their predicted amino acid sequences showed that katanin p60 belongs to the AAA family of ATPases and p80 is a WD40-containing protein. Katanin p60 exhibits MT-stimulated ATPase activity and MT severing activity in the absence of p80, although its MT severing activity can be stimulated by p80 (Hartman etal. 1998). It was demonstrated in an elegant fluorescence resonance energy transfer experiment that katanin p60 forms oligomers in an ATP- and MT-dependent manner and the assembly of p60 into oligomers is essential for its severing activity (Hartman and Vale 1999). Domain mapping experiments on the human katanin 060 have shown that the MT binding and severing activity is located at the N-terminal region of the protein, and the first 29 residues at the $\mathrm{N}$ terminus are required for its association with the p80 subunit (McNally et al. 2000; Figure 1E). Studies on katanin p80 have revealed that the WD40 domain is responsible for targeting of katanin to the centrosome (McNally et al. 1996) and the Cterminal half mediates interaction with p60 (Hartman et al. 1998; Figure 1F).

\section{Cellular Functions of Katanin in Animals}

The role of katanin in MT severing in vivo has been demonstrated in a number of studies. It was shown that in cultured vertebrate neurons, inhibition of katanin functions by microinjection of its antibodies led to a dramatic accumulation of MTs at the centrosome, indicating that the activity of katanin is required for the release of MTs from their nucleation sites in the centrosome (Ahmad et al. 1999). The same phenomenon was also observed by expression of dominant negative katanin p60 in rat fibroblasts and neurons, which resulted in an inhibition of MT release from the centrosome and an apparent increase in MT length (Karabay et al. 2004). In addition, transfection of cultured monkey kidney fibroblasts with dominant negative p60 prevented the redistribution of $\gamma$-tubulin from the centrosome to the mitotic spindles (Buster etal. 2002). Direct proof of MT severing by katanin in vivo was achieved by overexpression of human katanin p60 in HeLa cells, which caused abnormal fragmentation of interphase MTs (McNally etal. 2000). Overexpression of rat katanin p60 in cultured fibroblasts and neurons was also found to result in increased severing of MTs in vivo (Karabay etal. 2004). These studies demonstrate that katanin not only functions in the release of MTs from the nucleation sites but also severs MTs along their length.

An alteration in katanin activity has been found to cause various cellular defects. Katanin was shown to be concentrated at the mitotic and meiotic spindle poles in animal cells (McNally and Thomas 1998; Srayko etal. 2000). Loss-of-function mutation of the katanin homolog, $\mathrm{MEI}-1$, in $\mathrm{C}$. elegans resulted in disorganized meiotic spindles and meiotic failure, demonstrating that katanin is required for meiotic spindle organization (Srayko et al. 2000). It was shown that the katanin MT severing activity contributes to an increase in the total amount of MT polymers during meiotic spindle assembly (McNally et al. 2006; Srayko et al. 2006). The increased MT number was suggested to be a result of further polymerization of MT fragments generated by katanin severing. In addition to its functions in mitosis and meiosis, katanin plays important roles in animal cell growth. For example, alteration of katanin functions by expression of dominant negative p60 or overexpression of wild-type katanin p60 in cultured rat neurons leads to defective axon growth (Karabay et al. 2004).

\section{Identification of Katanin Homologs in Plants}

The first plant homolog of katanin p60 was identified and studied in Arabidopsis thaliana. A number of laboratories simultaneously investigated the functions of the Arabidopsis katanin p60, which is identified by various names, including AtKTN1 (Burk et al. 2001), AtKSS (McClinton et al. 2001), ERH3 (Webb et al. 2002), Atp60 (Stoppin-Mellet et al. 2002), LUE1 (Bouquin et al. 2003), 

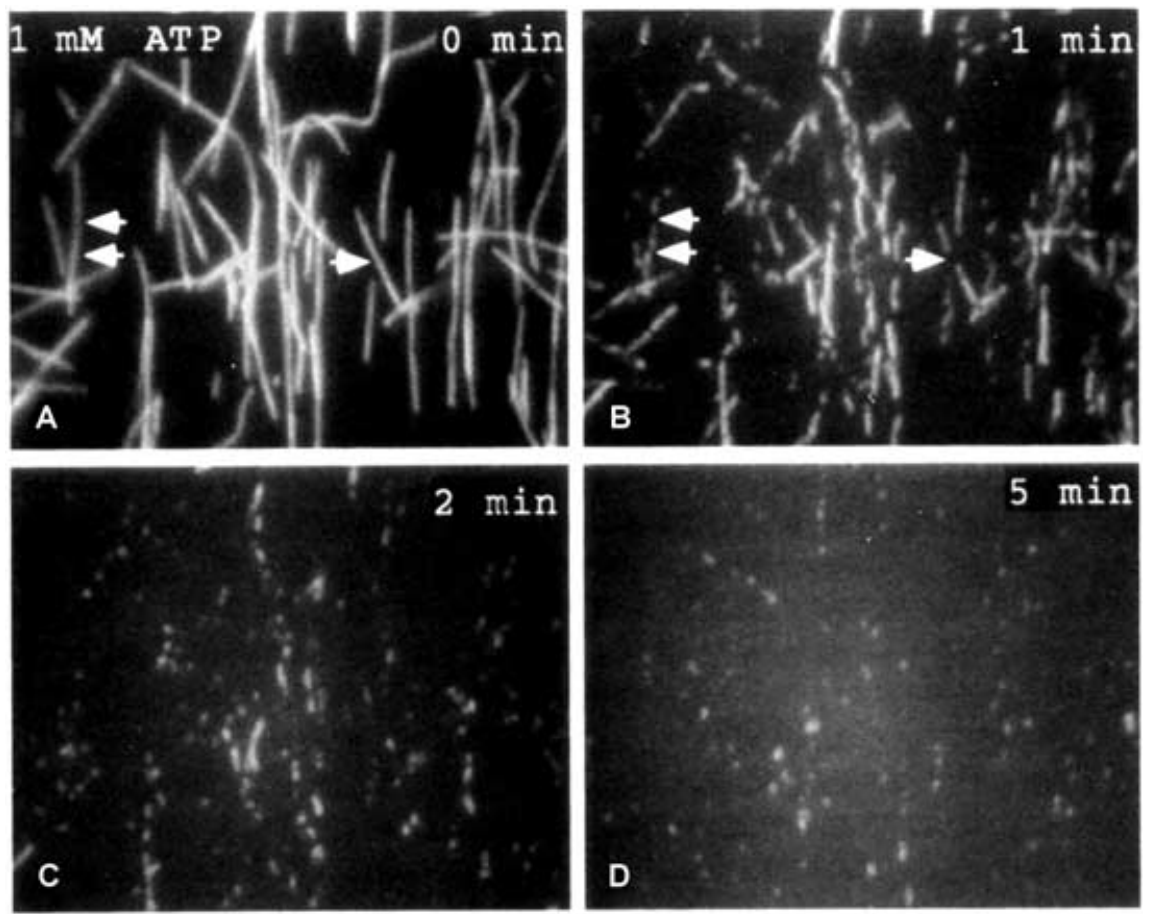

E

\section{Katanin p60}

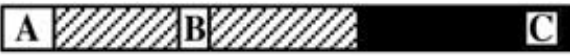

A p80-interacting domain

B MT-binding and severing domain

C ATPase domain

$\mathbf{F}$

\section{Katanin p80}

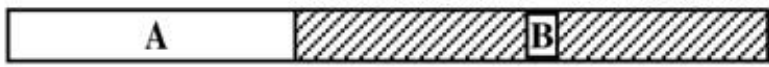
A WD40 domain involved in subcellular targeting
B p60-interacting domain

Figure 1. Fragmentation of MTs by the katanin MT severing protein.

(A-D) Visualization of MT severing before (A) and after (B to D) incubation of MTs with katanin purified from sea urchin at different time points. Taxol-stabilized, rhodamine-labeled MTs were immobilized on a glass coverslip and incubated with katanin in the presence of ATP. The same field of view of MTs was imaged and it is evident that individual MTs were progressively fragmented during incubation. Arrows indicate internal breaks in MTs. Reproduced from McNally and Vale (1993) with permission from the publisher.

(E) Diagram of the domain organization of katanin p60 subunit.

(F) Diagram of the domain organization of katanin p80 subunit. 


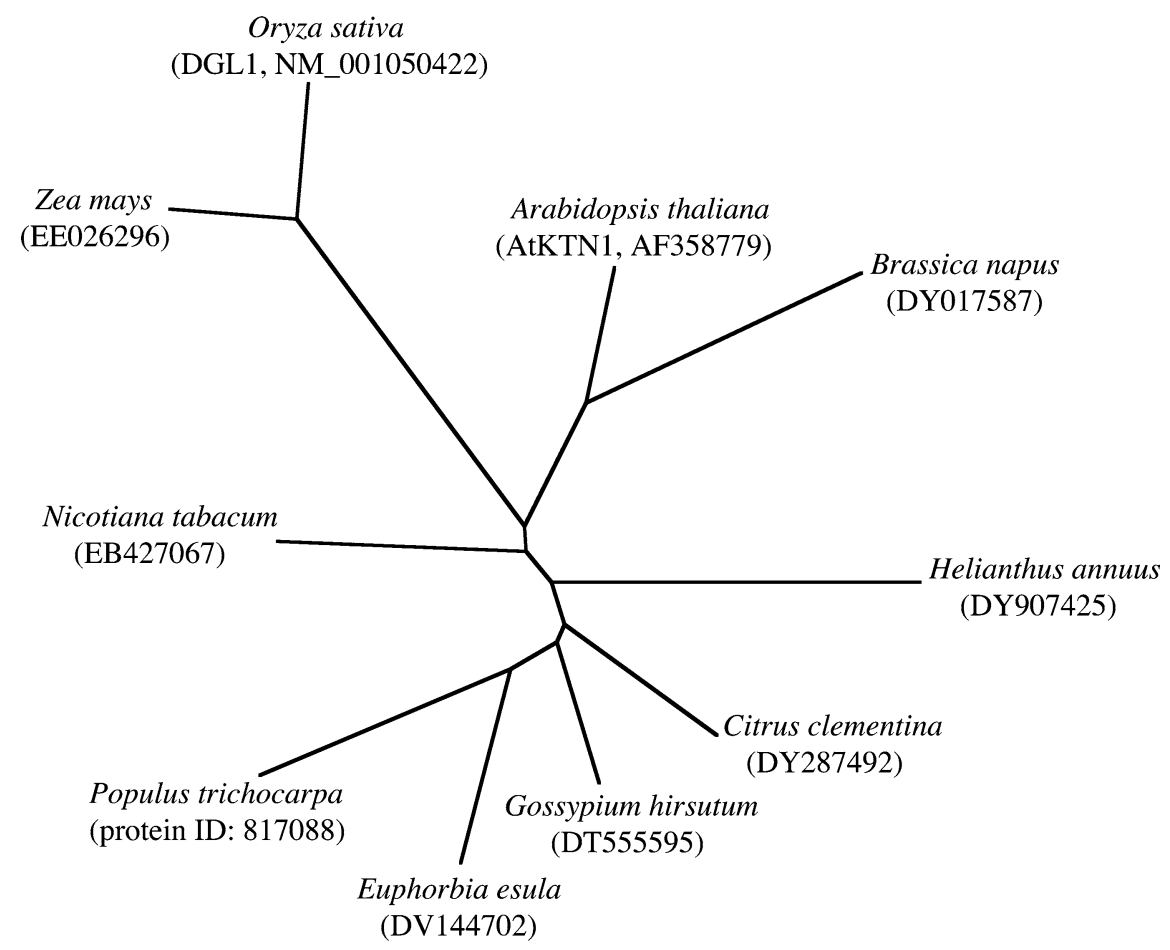

Figure 2. Phylogenetic tree of plant katanin homologs.

The N-terminal sequences containing the putative p80-interacting domain and the MT-binding and severing domain of plant katanin homologs were aligned using the ClustalW program (http://www.ebi.ac.uk/clustalw/), and the resulting alignment parameters were used to generate the phylogenetic tree using TREEVIEW (Page 1996). The sequence of the katanin homolog from Populus trichocarpa was retrieved from the DOE Joint Genome Institute website (http://genome.jgi-psf.org/Poptrl/Poptrl.home.html) and those from other plant species were obtained from the GenBank database and their accession numbers are shown in parentheses.

and BOTERO1 (Bichet etal. 2001). For simplicity, AtKTN1 is used throughout the text for the Arabidopsis katanin p60. AtKTN1 consists of 523 amino acid residues with a predicted molecular mass of $57 \mathrm{kD}$. It exhibits $43 \%$ identity and $56 \%$ similarity to sea urchin katanin p60. Recombinant AtKTN1 was shown to possess ATP-dependent MT severing activity in vitro, demonstrating that AtKTN1 is indeed a katanin MT severing protein (Burk and Ye 2002; Stoppin-Mellet et al. 2002). A BLAST search of the GenBank database using the MT-binding/severing domain sequence of AtKTN1 revealed the presence of katanin homologs in a number of plant species (Figure 2), suggesting an evolutionary conservation of katanin-mediated MT severing mechanisms in plants. This notion is supported by a study on the rice katanin p60 homolog DGL1 (Komorisono et al. 2005).

Although a single gene for katanin p60 exists in the Arabidopsis genome, four katanin p80 homologs (GenBank accession numbers: AAB71 474, CAC08 339, AAD4 999 and BAB09 559) were identified (Bouquin et al. 2003). Like sea urchin katanin p80, the Arabidopsis p80 homologs contain an N-terminal localized WD40 domain, which might be involved in targeting of katanin to certain subcellular locations. Except that one of the Arabidopsis katanin p80 homologs was shown to interact with AtKTN1 through its C-terminus (Bouquin et al. 2003), their cellular functions have not been elucidated.

\section{Katanin is Required for the Normal Organization of Cortical MTs in Plant Cells}

The localization of AtKTN1 has been investigated in several studies. Immunolocalization of AtKTN1 showed its localization in the perinuclear region and mitotic spindle poles (McClinton et al. 2001), and expression of GFP-tagged AtKTN1 in transgenic Arabidopsis plants revealed a punctate fluorescence pattern in elongating cells (Bouqin et al. 2003; Foster et al. 2003). A close examination of carrot protoplasts transfected with YFP-tagged AtKTN1 revealed that the punctate fluorescence signals are located at the cell cortex and appear to form an ordered pattern resembling that of cortical MTs (Figure 3), suggesting that AtKTN1 might be colocalized with cortical MTs. The additional punctate fluorescence signals seen in the cytoplasm are likely located at the perinuclear region (Figure 3D). 

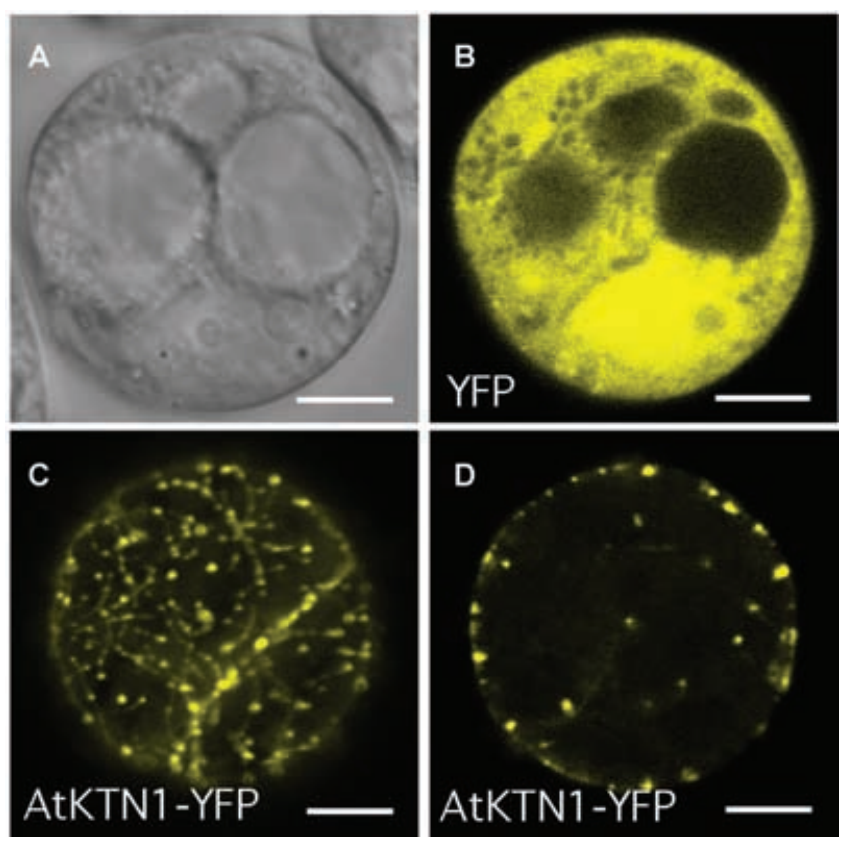

Figure 3. Subcellular localization of the Arabidopsis katanin AtKTN1.

Yellow fluorescent protein-tagged AtKTN1 was expressed in carrot protoplasts, and its fluorescence signals were visualized using a laser confocal microscope.

(A) Differential interference contrast image of a protoplast.

(B) A protoplast expressing YFP alone showing fluorescence signals throughout the cytoplasm and the nucleus.

(C, D) Surface (C) and mid-plane (D) views of a protoplast expressing AtKTN1-YFP, showing a punctate pattern of fluorescence signals in the cell cortex. Note that the ordered arrangement of fluorescence signals (C) resembles that of cortical MTs.

Mutations of the Arabidopsis AtKTN1 gene in the fra2/Bot1/leu1 mutants or of the rice katanin homolog DGL1 gene have been shown to result in disorganization of cortical MTs in elongating cells (Bichet et al. 2001; Burk et al. 2001; Burk et al. 2002; Bouquin et al. 2003; Komorisono et al. 2005). Many cortical MTs appear to converge at some common sites in the cortical region of fra2 mutant cells (Burk and Ye 2002). Because the nucleation sites for cortical MTs are known to be located at the cell cortex (Ehrhardt and Shaw 2006; Hashimoto and Kato 2006), the observed MT converging sites at the cortical region may be nucleation sites of cortical MTs and the fra2 mutation may cause a delay in the release of MTs from these sites. In addition to the aberrant organization of cortical MTs, the fra2 mutation also caused a delay in the disappearance of the perinuclear MT array in cells exited from cell division (Burk et al. 2001). In contrast to the roles of katanin in mitosis and meiosis in animals, AtKTN1 appears not to affect MT reorganization during mitosis and cytokinesis (Burk et al. 2001).
It was shown that in animal cells, katanin not only functions in the release of MTs from the nucleation sites but also severs MTs along their length. To investigate whether AtKTN1 can sever MTs along their length in vivo, we overexpressed the full-length AtKTN1 cDNA driven by the cauliflower mosaic virus $35 \mathrm{~S}$ promoter in Arabidopsis (Figure 4A). The AtKTN1 overexpressors exhibited a marked reduction in rosette size and inflorescence height, and a decrease in cell length and secondary wall thickness (Figure 4), a phenotype reminiscent of that of the fra2 mutant (Burk et al. 2001). However, overexpression of AtKTN1 did not affect root elongation, which is different from the fra2 mutant (Burk etal. 2001). It is interesting to note that the stems of AtKTN1 overexpressors are often cracked along their length (Figure 4C), which is likely a result of reduced cell wall strength. Surprisingly, examination of cortical MT organization revealed that the cortical MTs were not fragmented by AtKTN1 overexpression. Instead, they displayed an aberrant organization with many converging sites (Figure 5A to D), a phenotype similar to that seen in the fra2 mutant (Burk and Ye 2002). The observation that AtKTN1 overexpression does not cause fragmentation of cortical MTs in vivo suggests that the MT lattice is protected, probably by MT-associated proteins, from severing by katanin. There have been reports showing that expression of MT-associated proteins suppresses katanininduced MT severing in vivo in animal cells (Baas et al. 2005; Qiang et al. 2006). Nevertheless, overexpression of AtKTN1 led to an alteration in cortical MT organization, indicating that a tight regulation of AtKTN1 level is important for its normal cellular functions.

A recent study has shown that overexpression of RFP-tagged AtKTN1 causes random fragmentation and depolymerization of cortical MTs (Stoppin-Mellet etal. 2006). The fragmented MTs are often aggregated into thick bundles. This phenotype is apparently different from what we have described above. The difference could be due to the fact that Stoppin-Mellet et al. (2006) co-expressed GFP-tagged MT-binding domain of mammalian MAP4 in their overexpression study. The MAP4bound cortical MTs may not be protected by endogenous MTassociated proteins as are native cortical MTs, thus leading to their severing by katanin. Further studies are needed to clarify whether AtKTN1 can randomly sever MTs along their length in vivo.

\section{Katanin Plays Important Roles in Cellulose Microfibril Deposition and Cell Morphogenesis in Plants}

Studies of katanin mutants in Arabidopsis and rice established important roles of katanin in the oriented deposition of cellulose microfibrils, cell wall biosynthesis, cell morphogenesis, and hormonal responses. The Arabidopsis fra2 mutation has been shown to cause an aberrant orientation of cellulose microfibrils 

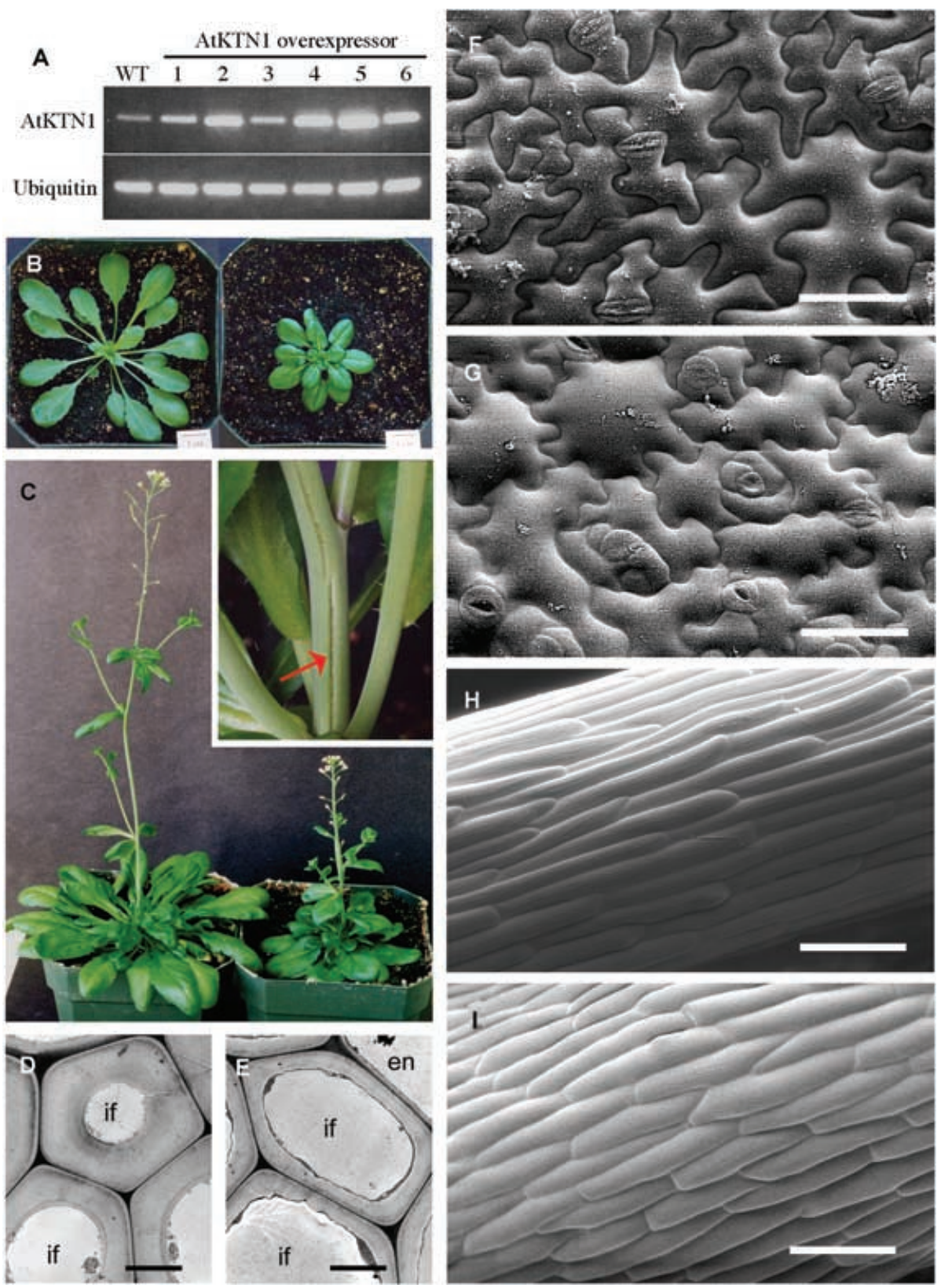

Figure 4. Excess AtKTN1 causes a reduction in the expansion/elongation of cells and organs and a decrease in cell wall strength.

(A) RT-PCR analysis showing an elevated level of AtKTN1 transcript in the AtKTN1 overexpressors (upper panel). The expression of a ubiquitin gene was used as an internal control (lower panel). Transgenic line \#5 was used for further phenotypic characterization.

(B) Three-week-old plants showing an AtKTN1 overexpressor (right) with a drastic reduction in rosette size compared with the wild type (left).

(C) Six-week-old plants showing an AtKTN1 overexpressor (right) with a much shorter inflorescence stem than the wild type (left). Inset shows the abnormal cracks (arrow) along the stem of AtKTN overexpressors, which is likely due to reduced cell wall strength.

(D, E) Transmission electron micrographs of interfascicular fibers showing a severe decrease in secondary wall thickness in the AtKTN1 overexpressors (E) compared with the wild type (D). en, endodermis; if, interfascicular fiber. Bars, $2.5 \mu \mathrm{m}$.

(F, G) Scanning electron micrographs of the abaxial leaf surfaces showing the presence of fewer lobes in ordinary epidermal cells of the AtKTN1 overexpressors (G) compared with the wild type (F). Bars, $50 \mu \mathrm{m}$.

(H, I) Scanning electron micrographs of the epidermis of stamen filaments showing the reduced cell length in the AtKTN1 overexpressors (I) compared with the wild type $\mathbf{( H )}$. Bars, $50 \mu \mathrm{m}$. 

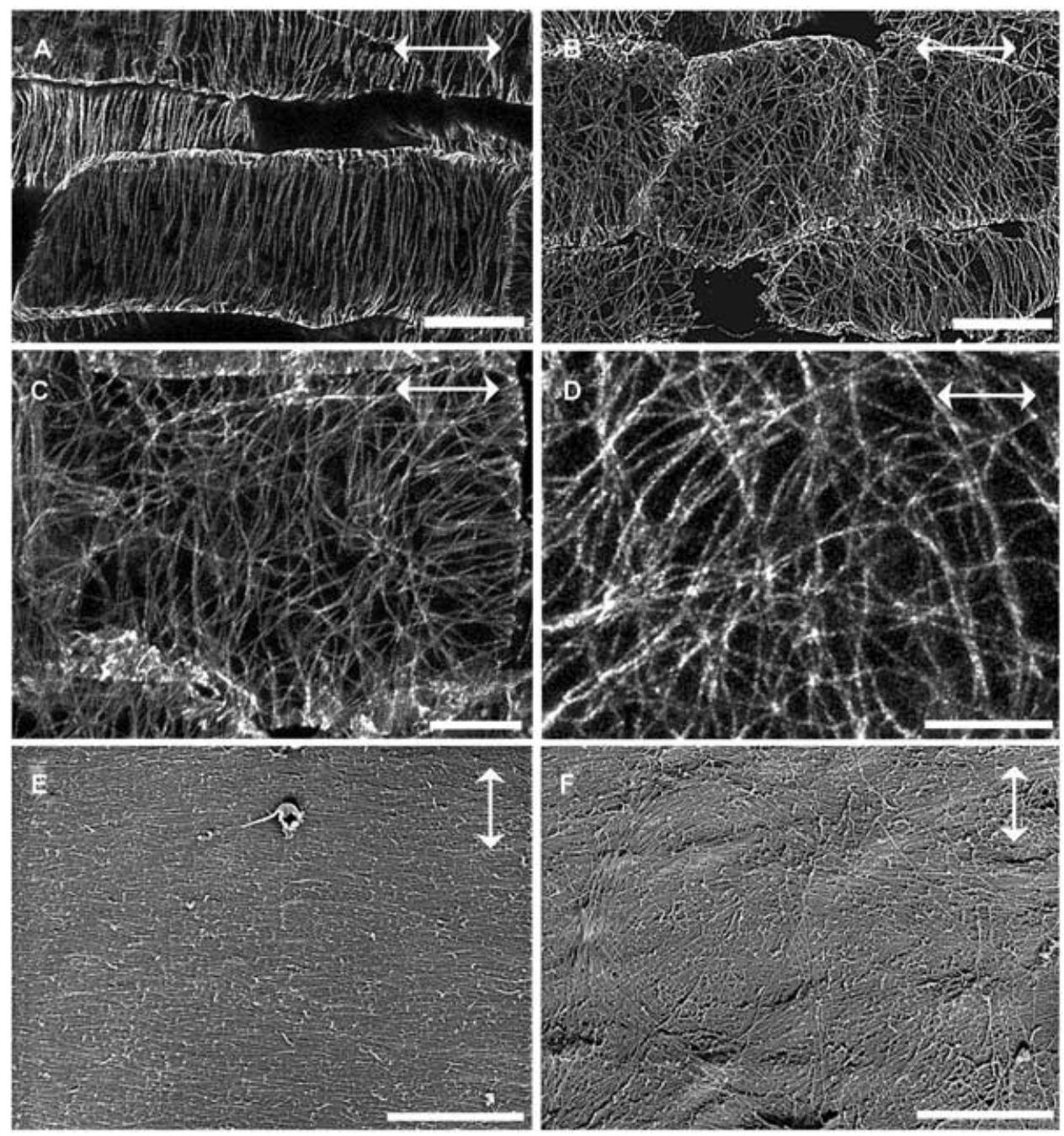

Figure 5. Excess AtKTN1 results in disorganization of cortical MTs and aberrant deposition of cellulose microfibrils.

For detection of MTs, pith cells from elongating stem internodes were used for MT labeling with a monoclonal antibody against $\alpha$-tubulin. Antibodylabeled MTs were detected with fluorescein isothiocyanate-conjugated secondary antibodies, and the signals were visualized with a laser confocal microscope. For visualization of cellulose microfibrils, elongating stem internodes were longitudinally sectioned, and the cellulose microfibrils in the innermost layer of cell walls of pith cells were observed using field emission scanning electron microscopy. Double-headed arrows indicate the elongation axis. Bars, $20 \mu \mathrm{m}$ in (A, B); $10 \mu \mathrm{m}$ in (C, D); $1 \mu \mathrm{m}$ in (E, F).

(A) Wild-type pith cells showing transversely oriented cortical MTs.

(B) Pith cells of an AtKTN1 overexpressor showing disorganized cortical MTs.

(C, D) Higher magnification of cortical MTs from (B) showing the aberrantly arranged MTs with multiple sites of MT convergence.

(E) Cellulose microfibrils in a wild-type pith cell showing their transverse orientation.

(F) Cellulose microfibrils in a pith cell of AtKTN1 overexpressors showing a dramatic change in their orientation.

in both elongating parenchyma cells and in fibers undergoing secondary wall thickening (Burk and Ye 2002). Because cortical MTs are believed to provide tracks for the oriented deposition of cellulose microfibrils (Baskin 2001), the aberrant deposition of cellulose microfibrils seen in the fra 2 mutant is likely a result of the disrupted organization of cortical MTs. The disorgani- zation of cortical MTs and/or aberrant deposition of cellulose microfibrils in the fra2 mutant may impede the normal cell wall biosynthesis, thus resulting in reduced wall thickness in both parenchyma cells and fibers (Burk and Ye 2002).

The most prominent phenotype of the katanin mutants is the reduced cell elongation and consequent decrease in 
organ length/height (Bichet et al. 2001; Burk et al. 2001; Webb et al. 2002; Bouquin et al. 2003; Komorisono et al. 2005). This could be attributed to the defective cortical MT organization and/or aberrant cellulose microfibril deposition, both of which are known to be important for normal cell morphogenesis. An alteration in hormonal responses and in the expression of gebberellin (GA) biosynthetic genes was also observed in the katanin mutants (Bouqin et al. 2003; Komorisono et al. 2005).

\section{Katanin Homologs in Algae}

In addition to its essential functions in animals and plants, katanin was also found to play important roles in algae. The flagella of the alga Chlamydomonas reinhardtii are complex macromolecular assemblies in which the central apparatus is composed of two singlet MTs. Dymek et al. (2004) showed that mutation of a katanin $\mathrm{p} 80$ homolog caused a complete loss of the central pair of MTs, leading to paralyzed flagella. This finding suggests a novel role of katanin p80 in the assembly and/or stability of the central pair of flagellar MTs. A katanin p60 homolog was also identified in Chlamydomonas reinhardtii and it was suggested to mediate the severing of MTs during deflagellation (Lohret et al. 1998, 1999).

\section{Perspectives}

The findings that plant katanin plays important roles in cortical MT organization and cell morphogenesis provide new insights into our understanding of katanin functions. Although it is clear that katanin is required for the normal organization of cortical MTs in plant cells, the underlying mechanism by which katanin regulates cortical MT organization is not known. In animals, it has been demonstrated that katanin functions in the release of MTs from their nucleation sites as well as in severing MTs into smaller fragments, which serve as templates for further polymerization of MTs. It is likely that katanin performs similar functions in plants. Because cortical MTs are nucleated at the cell cortex, it is conceivable that katanin is involved in the release of MTs from their nucleation sites at the cell cortex. The released MTs then undergo reorganization at the cortical region to form ordered patterns. This hypothesis is supported by the observations that many cortical MTs in the katanin mutants appear to converge at some common sites, possibly MT nucleation sites, and that katanin is localized at the cell cortex in a punctate pattern, which may correspond to cortical MT nucleation sites. Whether the release of cortical MTs from their nucleation sites is indeed mediated by katanin remains to be investigated. Because there are four katanin p80 homologs in the Arabidopsis genome and one of them interacts with AtKTN1, it is likely that these p80 homologs are involved in the targeting of katanin to specific locations such as cell cortex, perinuclear envelope and mitotic spindles. Further investigation of the functions of katanin $\mathrm{p} 80$ homologs in plants is expected to further our understanding of how katanin is targeted to different subcellular locations to exert its functions.

\section{References}

Ahmad FJ, Yu W, McNally FJ, Baas PW (1999). An essential role for katanin in severing microtubules in the neuron. J. Cell Biol. 145, 305-315.

Baas PW, Karabay A, Qiang L (2005). Microtubules cut and run. Trends Cell Biol. 15, 518-524.

Baskin TI (2001). On the alignment of cellulose microfibrils by cortical microtubules: a review and a model. Protoplasma 215, 150-171.

Bichet A, Desnos T, Turner S, Grandjean O, Hofte H (2001). $B O T E R O 1$ is required for normal orientation of cortical microtubules and anisotropic cell expansion in Arabidopsis. Plant J. 25, 137-148.

Bouquin T, Mattsson O, Naested H, Foster R, Mundy J (2003). The Arabidopsis lue1 mutant defines a katanin p60 ortholog involved in hormonal control of microtubule orientation during cell growth. J. Cell Sci. 116, 791-801.

Burk DH, Liu B, Zhong R, Morrison WH, Ye ZH (2001). A katanin-like protein regulates normal cell wall biosynthesis and cell elongation. Plant Cell 13, 807-827.

Burk DH, Ye ZH (2002). Alteration of oriented deposition of cellulose microfibrils by mutation of a katanin-like microtubule-severing protein. Plant Cell 14, 2145-2160.

Buster D, McNally K, McNally FJ (2002). Katanin inhibition prevents the redistribution of gamma-tubulin at mitosis. J. Cell Sci. 115, 10831092.

Dymek EE, Lefebvre PA, Smith EF (2004). PF15p is the Chlamydomonas homologue of the katanin p80 subunit and is required for assembly of flagellar central microtubules. Eukaryot. Cell 3, 870879.

Ehrhardt DW, Shaw SL (2006). Microtubule dynamics and organization in the plant cortical array. Annu. Rev. Plant Biol. 57, 859-875.

Foster R, Mattsson O, Mundy J (2003). Plants flex their skeletons. Trends Plant Sci. 8, 202-204.

Hartman JJ, Mahr J, McNally K, Okawa K, Iwamatsu A, Thomas S et al. (1998). Katanin, a microtubule-severing protein, is a novel AAA ATPase that targets to the centrosome using a WD40-containing subunit. Cell 93, 277-287.

Hartman JJ, Vale RD (1999). Microtubule disassembly by ATPdependent oligomerization of the AAA enzyme katanin. Science 286, 782-785.

Hishimoto T, Kato T (2006). Cortical control of plant microtubules. Curr. Opin. Plant Biol. 9, 5-11.

Karabay A, Yu W, Solowska JM, Baird DH, Baas PW (2004). Axonal growth is sensitive to the levels of katanin, a protein that severs microtubules. J. Neurosci. 24, 5778-5788.

Komorisono M, Ueguchi-Tanaka M, Aichi I, Hasegawa Y, Ashikari $\mathbf{M}, \mathbf{K i t a n o} \mathbf{H}$ et al. (2005). Analysis of the rice mutant $d w a r f$ and gladius leaf 1. Aberrant katanin-mediated microtubule organization 
causes up-regulation of gibberellin biosynthetic genes independently of gibberellin signaling. Plant Physiol. 138, 1982-1993.

Lohret TA, McNally FJ, Quarmby LM (1998). A role for kataninmediated axonemal severing during Chlamydomonas deflagellation. Mol. Biol. Cell 9, 1195-1207.

Lohret TA, Zhao L, Quarmby LM (1999). Cloning of Chlamydomonas p60 katanin and localization to the site of outer doublet severing during deflagellation. Cell Motil. Cytoskeleton 43, 221-231.

McClinton RS, Chandler JS, Callis J (2001). cDNA isolation, characterization, and protein intracellular localization of a katanin-like p60 subunit from Arabidopsis thaliana. Protoplasma 216, 181-190.

McNally K, Audhya A, Oegema K, McNally FJ (2006). Katanin controls mitotic and meiotic spindle length. J. Cell Biol. 175, 881-891.

McNally KP, Bazirgan OA, McNally FJ (2000). Two domains of p80 katanin regulate microtubule severing and spindle pole targeting by p60 katanin. J. Cell Sci. 113, 1623-1633.

McNally FJ, Okawa K, Iwamatsu A, Vale RD (1996). Katanin, the microtubule-severing ATPase, is concentrated at centrosomes. $J$. Cell Sci. 109, 561-567.

McNally FJ, Thomas S (1998). Katanin is responsible for the M-phase microtubule-severing activity in Xenopus eggs. Mol. Biol. Cell $\mathbf{9}$, 1847-1861.

McNally FJ, Vale RD (1993). Identification of katanin, an ATPase that severs and disassembles stable microtubules. Cell 75, 419-429.
Page RDM (1996). TREEVIEW: An application to display phylogenetic trees on personal computers. CABIOS 12, 357-358.

Qiang L, Yu W, Andreadis A, Luo M, Baas PW (2006). Tau protects microtubules in the axon from severing by katanin. J. Neurosci. 26, 3120-3129.

Srayko M, Buster DW, Bazirgan OA, McNally FJ, Mains PE (2000). MEl-1/MEl-2 katanin-like microtubule severing activity is required for Caenorhabditis elegans meiosis. Genes Dev. 14, 10721084.

Srayko M, O'toole ET, Hyman AA, Muller-Reichert T (2006). Katanin disrupts the microtubule lattice and increases polymer number in C. elegans meiosis. Curr. Biol. 16, 1944-1949.

Stoppin-Mellet V, Gaillard J, Vantard M (2002). Functional evidence for in vitro microtubule severing by the plant katanin homologue. Biochem. J. 365, 337-342.

Stoppin-Mellet V, Gaillard J, Vantard M (2006). Katanin's severing activity favors bundling of cortical microtubules in plants. Plant J. 46, 1009-1017.

Vale RD (1991). Severing of stable microtubules by a mitotically activated protein in Xenopus egg extracts. Cell 64, 827-839.

Webb M, Jouannic S, Foreman J, Linstead P, Dolan L (2002). Cell specification in the Arabidopsis root epidermis requires the activity of ECTOPIC ROOT HAIR 3-a katanin-p60 protein. Development 129, 123-131. 